
$\$$ Research Square
Preprints are preliminary reports that have not undergone peer review.
They should not be considered conclusive, used to inform clinical practice, or referenced by the media as validated information.

\title{
[18F]2-fluoro-2-deoxy-sorbitol PET Imaging for Quantitative Estimation of Blood-brain Barrier Permeability in vivo
}

\section{Gaelle Hugon}

Universite Paris-Saclay

Sébastien Goutal

Université Paris-Saclay

Ambre Dauba

Universite Paris-Saclay

Louise Breuil

Universite Paris-Saclay

Alexandra Winkeler

Universite Paris-Saclay

Anthony Novell

Universite Paris-Saclay

Nicolas Tournier ( $\square$ n.tournier@universite-paris-saclay.fr )

Université Paris-Saclay https://orcid.org/0000-0002-0755-2030

\section{Short Report}

Keywords: Blood-brain barrier, integrity marker, sorbitol, positron emission tomography

Posted Date: July 13th, 2021

DOl: https://doi.org/10.21203/rs.3.rs-693599/v1

License: (c) (i) This work is licensed under a Creative Commons Attribution 4.0 International License. Read Full License 


\section{Abstract}

Purpose The non-transported and non-metabolized sorbitol derivative $\left[{ }^{18} \mathrm{~F}\right] 2$-fluoro-2-deoxy-sorbitol $\left(\left[{ }^{18} \mathrm{~F}\right] \mathrm{FDS}\right)$ can be straightforwardly obtained from chemical reduction of commercial $\left[{ }^{18} \mathrm{~F}\right] 2$-deoxy-2fluoro-D-glucose. $\left[{ }^{18} \mathrm{~F}\right] \mathrm{FDS}$ was evaluated as a small-molecule (paracellular) marker of blood-brain barrier (BBB) integrity for PET.

Methods Five mice underwent focused ultrasound (FUS) to generate spatially controlled BBB disruption in the right hemisphere. PET kinetics of $\left[{ }^{18} \mathrm{~F}\right] \mathrm{FDS}$ in each brain hemisphere were described by a 1-tissue compartment model using an image-derived input function.

Results BBB disruption resulted in a $2.4 \pm 0.8$-fold increase in the brain distribution $\left(V_{\mathrm{T}} \mathrm{p}<0.01\right)$ of $\left[{ }^{18} \mathrm{~F}\right] \mathrm{FDS}$. Enhanced brain uptake was associated with an increase in the influx transfer rate $K_{1}(+1.4 \pm 0.7$-fold, $\mathrm{p}<0.05)$ and a decrease in the efflux transfer rate $k_{2}(-1.7 \pm 0.4$-fold, $\mathrm{p}<0.01)$.

Conclusion Thanks to the quantitative performance of PET compared with other neuroimaging techniques, $\left[{ }^{18} \mathrm{~F}\right]$ FDS PET and kinetic modelling provides a readily available and sensitive method for non-invasive determination of different levels of BBB permeability in vivo.

\section{Introduction}

The blood-brain barrier (BBB) plays a critical role in protecting the brain from the circulation while controlling brain homeostasis. Integrity of the BBB is mainly carried out by tight junctions between adjacent endothelial cells forming the brain microvasculature [1]. A large body of translational research supports that BBB integrity is compromised in many CNS pathological conditions including multiple sclerosis, hypoxic/ischemic insult, traumatic injury, Parkinson's and Alzheimer's diseases, epilepsy and brain tumors [2]. Different non-clinical models have shown that BBB leakage is a common feature of the neuroinflammatory cascade and contributes to disease-associated brain damage $[3,4]$.

Quantitative markers are needed to demonstrate loss in BBB integrity in various pathophysiological conditions [5]. In non-clinical in vitro and ex vivo research, low molecular weight (MW, g/mol) hydrophilic molecules such as fluorescein ( $\mathrm{MW}=332$, fluorescent detection), (radio)labeled analogues of sucrose (MW = 342) or mannitol (MW = 182) are preferred as quantitative "paracellular" (i.e between-cell pathway) markers of membrane integrity (Fig. 1) [2, 6]. Low MW is associated with enhanced sensitivity to subtle change in barrier permeability compared with higher MW compounds such as Evan's blue (EB, MW $=961$, highly bound to plasma proteins), radiolabeled proteins (albumin MW $=65,000)$, dextrans $(M W=1,500-$ $70,000)$ or inulin $(\mathrm{MW}=6179)[2,5]$.

Imaging techniques enable in vivo determination of BBB integrity with translational perspectives. Dynamic contrast-enhanced (DCE) and dynamic susceptibility contrast MRI using gadolinium (Gd)-based contrast agents such as gadoterate $(M W=558)$ or Gd-DTPA (Gd-diethylene triamine pentaacetic acid, 
$M W=546)[7]$, and brain SPECT using [ ${ }^{99 \mathrm{~m}} \mathrm{Tc}$ ]DTPA $(\mathrm{MW}=487)$ are widely used to detect local BBB disruption associated with some situations such as ischemic stroke or brain tumors [8]. Despite limited quantitative performance, the predominant use of MRI and SPECT to investigate BBB integrity is likely due to the availability of corresponding imaging probes [9]. PET may allow for accurate kinetic modeling and quantitative estimation of the transfer of radiolabeled compounds across the BBB in vivo[10]. However, PET using radiolabeled low- or high-MW makers of BBB integrity such as $\left[{ }^{18} \mathrm{~F}\right] 1$-fluoro-1-deoxyD-mannitol $(M W=183)[11]$ or $\left[{ }^{11} \mathrm{C}\right]$ inulin $(M W=6179)$ [12] did not reach mainstream use, probably due to their limited availability in centers not equipped with a cyclotron.

Sorbitol, a stereoisomer of mannitol, is a non-transported hydrophilic small molecule $(M W=182)$ that is poorly metabolized in mammals. The fluorinated derivative $\left[{ }^{18} \mathrm{~F}\right] 2$-fluoro-2-deoxy-sorbitol $\left(\left[{ }^{18} \mathrm{~F}\right] \mathrm{FDS}, \mathrm{MW}=\right.$ 183) can be virtually obtained in all Nuclear Medicine departments from simple chemical reduction of commercial $\left[{ }^{18} \mathrm{~F}\right] 2$-fluoro-2-deoxy-D-glucose ( $\left[{ }^{18} \mathrm{~F}\right] \mathrm{FDG}$, Fig. 1) [13]. $\left[{ }^{18} \mathrm{~F}\right] \mathrm{FDS}$ shows non-significant brain uptake in healthy rodents and humans [14] and benefits from favorable pharmacokinetic properties for quantitative PET imaging in vivo [15].

This study aimed at evaluating $\left[{ }^{18} \mathrm{~F}\right] \mathrm{FDS}$ PET and kinetic modelling for quantitative determination of BBB permeability in vivo. To this end, a method based on focused ultrasound (FUS) was optimized to induce hemispheric BBB disruption in mice.

\section{Material And Methods}

\section{Production of $\left[{ }^{18} \mathrm{~F}\right] \mathrm{FDS}$}

Synthesis of $\left[{ }^{18} \mathrm{~F}\right] \mathrm{FDS}$ from commercial $\left[{ }^{18} \mathrm{~F}\right] \mathrm{FDG}$ and quality control was described by Li et al. [13] and is reported as Supplementary material.

\section{Focused Ultrasound}

The method for spatially controlled BBB disruption was optimized from previous work [16] to induce reproducible BBB disruption in the right brain hemisphere only. Seven-week-old female NMRI nu/nu mice were anesthetized with $1.5 \%$ isoflurane in $\mathrm{O}_{2}$. A catheter was inserted in the tail vein and the animal was transferred to the sonication system. Microbubbles $(50 \mu \mathrm{L})$ were intravenously administrated in the tail vein before the beginning of the FUS ( $n=5)$ or Sham (no FUS, $n=3$ ) session. Detailed FUS protocol is reported as Supplementary material.

\section{Evan's blue extravasation test}

Evan's blue (EB) (Sigma-Aldrich, Saint-Quentin Fallavier, France) at $4 \%$ in $\mathrm{NaCl} 0.9 \%$ was freshly prepared as previously described [17]. Mice received $100 \mu \mathrm{L}$ EB i.v immediately after FUS. One hour after injection, i.e. at the end of PET acquisition, animals were euthanized and brains were removed to visually assess EB extravasation (Fig. 2). 


\section{$\left[{ }^{18} \mathrm{~F}\right] \mathrm{FDS}$ PET imaging}

Immediately after EB injection, anesthetized mice were transferred to the microPET scanner (Inveon, microPET, Siemens Healthcare, Knoxville, TN, USA). $\left[{ }^{18} \mathrm{~F}\right] \mathrm{FDS}$ was administered intravenously $(4.2 \pm 0.76$ $\mathrm{MBq}$ ) using a microinjection pump at the rate of $0.2 \mathrm{~mL}^{\mathrm{min}} \mathrm{m}^{-1}(\mathrm{n}=5 \mathrm{FUS} ; \mathrm{n}=3$ Sham). Dynamic PET acquisition (60 $\mathrm{min})$ started with $\left[{ }^{18} \mathrm{~F}\right] \mathrm{FDS}$ injection.

PET images were reconstructed by the 3D OSEM/MAP algorithm and corrected for attenuation, random coincidences and scatter. Volumes of interests $\left(\mathrm{VOI}_{\mathrm{s}}\right)$ were manually delimited using Pmod software (version 3.8, PMOD Technologies Ltd, Zurich, Switzerland). In the FUS group, extravasation of ${ }^{18} \mathrm{~F}$-FDS was obvious in the sonicated area on late PET images (Fig. 2A). The region with disrupted BBB was delineated and mirrored to the contralateral hemisphere. In Sham animals, $\mathrm{VOI}_{\mathrm{s}}$ were drawn in each brain hemisphere. Another VOI was drawn on the aorta (blood-pool), obvious on early time-frames, to generate an image derived input function (IDIF).

Time Activity Curves (TACs) were corrected for radioactive decay and expressed as standard uptake value (SUV) vs time. Area Under the TAC (AUC) was calculated from 0 to 60 min. A 1-tissue compartment (1-TC) model using IDIF was tested to describe the transport of ${ }^{18} \mathrm{~F}$-FDS across the BBB and generate parametric mapping of the total volume of distribution $\left(V_{T}\right)$ of $\left[{ }^{18} \mathrm{~F}\right] \mathrm{FDS}$ (Fig. 2B). Data are reported as mean \pm standard deviation (S.D.) and were statistically compared using either a One-way ANOVA or a paired ttest. (GraphPad Prism, La Jolla, CA, USA).

\section{Results}

In hemispheres with intact BBB (Sham animals or contralateral hemisphere), brain PET signal increased rapidly with maximal uptake at $T_{\max } \sim 2.0 \pm 0.9 \mathrm{~min}$. FUS enhanced the PET signal in the sonicated volume consistent with EB extravasation (Fig. 2) and $T_{\max }$ was achieved later at $5.5 \pm 2.3 \mathrm{~min}$. Brain uptake of $\left[{ }^{18} \mathrm{~F}\right] \mathrm{FDS}$ in the sonicated brain was significantly higher compared with the contralateral area $(2.12 \pm 1.5$-fold increase, $p<0.01)$ or the Sham group $(1.84 \pm 1.09$-fold increase, $p<0.05)$ (Fig. 3$)$.

Brain TACs were accurately described by the 1-TC model which demonstrated a significant $2.43 \pm 0.8$-fold increase in the $V_{T}$ of $\left[{ }^{18} \mathrm{~F}\right] \mathrm{FDS}$ in the sonicated area $(\mathrm{p}<0.001)$. Enhanced brain distribution was associated with a $1.4 \pm 0.7$-fold increase in $K_{1}(p<0.05)$ and a $1.7 \pm 0.4$-fold decrease in $k_{2}(p<0.01)$ (Fig. 4).

\section{Discussion}

$\left[{ }^{18} \mathrm{~F}\right] \mathrm{FDS}$ benefits from the characteristics of an "ideal" marker of BBB integrity [5]. This includes clinical safety, metabolic stability, low binding to plasma proteins $(<0.1 \%)$ and low baseline brain uptake when 
the BBB is intact $[14,18]$. Simple production of ${ }^{18} \mathrm{~F}$-FDS from commercial $\left[{ }^{18} \mathrm{~F}\right] \mathrm{FDG}$ makes it an appealing radiopharmaceutical candidate for determination of BBB integrity using quantitative PET.

Unlike $\left[{ }^{18} \mathrm{~F}\right] \mathrm{FDG},\left[{ }^{18} \mathrm{~F}\right] \mathrm{FDS}$ is poorly taken up by mammalian cells because it does not undergo facilitated transport [13]. $\left[{ }^{18} \mathrm{~F}\right.$ FDS PET has been validated in animals and humans to study renal function [15] or detect/estimate bacterial burden in tissues because sorbitol is a specific metabolic substrate of some strains of gram-negative bacteria [19]. Interestingly, $\left[{ }^{18} \mathrm{~F}\right] \mathrm{FDS}$ was shown to visually accumulate in orthotopic brain tumor xenografts in mice, despite negligible uptake by implanted glioma cells in vitro [13]. Our results suggest that enhanced $\left[{ }^{18} \mathrm{~F}\right]$ FDS PET signal in the tumor area may be attributed to local BBB leakage.

Pharmacokinetic modelling of brain $\left[{ }^{18} \mathrm{~F}\right] \mathrm{FDS}$ PET data is relatively simple. The impact of BBB disruption did not restrict to enhanced influx (blood-to-brain) transfer. This suggests that considering the uptake phase of the brain distribution only may underestimate the overall impact of BBB integrity on brain exposure of solutes.

FUS offers unique perspectives to induce safe, reproducible and localized BBB disruption in vivo [20]. Interestingly, mapping of $\left[{ }^{18} \mathrm{~F}\right] F D S$ brain distribution within the sonicated area displayed a gradient from the center to the periphery (Fig. 2). It may be hypothesized that heterogeneity in intensity of delivered ultrasound may occur within the sonicated area as a consequence of loss of ultrasound transmission relative to the transducer angulation at the skull surface. Interestingly, such phenomenon could not be detected using high-MW markers of BBB integrity such as gadoterate ou EB [17].

There is a critical need for PET imaging markers to offer more quantitative insight into BBB integrity in pathophysiological conditions [11, 21-23]. Outcome parameters and parametric images describing BBB transport of $\left[{ }^{18} \mathrm{~F}\right] \mathrm{FDS}$ provide absolute quantitative whole-brain mapping of BBB permeability. This may be useful for group comparison or to investigate the dynamics of disease-associated change in BBB permeability in longitudinal studies. In a neuropharmacokinetic perspective, $\left[{ }^{18} \mathrm{~F}\right] \mathrm{FDS}$ provides a convenient alternative to EB, DCE-MRI or [ $\left.{ }^{99 \mathrm{~m}} \mathrm{TC}\right] \mathrm{DTPA}$ SPECT, with better quantitative performance, to interpret the brain penetration of CNS-targeting radioligands with respect to the local permeability of the BBB [24].

\section{Declarations}

\section{Funding}

Gaelle Hugon received a PhD grant from the CEA. Louise Breuil received a grant from CEA/AP-HP.

\section{Conflicts of interest/Competing interests}

None. 


\section{Availability of data and material}

All data are available upon reasonable request to the corresponding author.

\section{Code availability}

N.A

\section{Authors' contributions}

All authors contributed to the study conception and design. Material preparation, data collection and analysis were performed by Gaëlle Hugon, Sébastien Goutal, Ambre Dauba, Louise Breuil, Alexandra Winkeler, Anthony Novell and Nicolas Tournier. The first draft of the manuscript was written by Gaëlle Hugon and all authors commented on previous versions of the manuscript. All authors read and approved the final manuscript.

\section{Ethics approval}

All animal experiments were performed in accordance with the recommendations of the European Community (2010/63/UE) and the French National Committees (law 2013-118) for the care and use of laboratory animals. The experimental protocol was approved by a local ethics committee for animal use (CETEA) and by the French ministry of agriculture (APAFIS 16292-2018072609593031 v7).

\section{Acknowledgments}

We thank Maud Goislard for helpful technical assistance.

\section{References}

1. Abbott NJ, Patabendige AAK, Dolman DEM, Yusof SR, Begley DJ. Structure and function of the blood-brain barrier. Neurobiol Dis. 2010;37:13-25.

2. Kadry H, Noorani B, Cucullo L. A blood-brain barrier overview on structure, function, impairment, and biomarkers of integrity. Fluids Barriers CNS. 2020;17:69.

3. Obermeier B, Daneman R, Ransohoff RM. Development, maintenance and disruption of the bloodbrain barrier. Nat Med. 2013;19:1584-96.

4. Haruwaka K, Ikegami A, Tachibana Y, Ohno N, Konishi H, Hashimoto A, et al. Dual microglia effects on blood brain barrier permeability induced by systemic inflammation. Nat Commun. Nature Publishing Group; 2019;10:5816.

5. Saunders NR, Dziegielewska KM, Møllgård K, Habgood MD. Markers for blood-brain barrier integrity: how appropriate is Evans blue in the twenty-first century and what are the alternatives? Front Neurosci; 2015: https://www.frontiersin.org/articles/10.3389/fnins.2015.00385/full 
6. Noorani B, Chowdhury EA, Alqahtani F, Ahn Y, Patel D, Al-Ahmad A, et al. LC-MS/MS-based in vitro and in vivo investigation of blood-brain barrier integrity by simultaneous quantitation of mannitol and sucrose. Fluids Barriers CNS. 2020;17:61.

7. Elschot EP, Backes WH, Postma AA, van Oostenbrugge RJ, Staals J, Rouhl RPW, et al. A Comprehensive View on MRI Techniques for Imaging Blood-Brain Barrier Integrity. Invest Radiol. 2021;56:10-9.

8. Kassner A, Thornhill R. Measuring the integrity of the human blood-brain barrier using magnetic resonance imaging. Methods Mol Biol. 2011;686:229-45.

9. Raja R, Rosenberg GA, Caprihan A. MRI measurements of Blood-Brain Barrier function in dementia: A review of recent studies. Neuropharmacology. 2018;134:259-71.

10. Tournier N, Comtat C, Lebon V, Gennisson J-L. Challenges and Perspectives of the Hybridization of PET with Functional MRI or Ultrasound for Neuroimaging. Neuroscience. https://doi.org/10.1016/j.neuroscience.2020.10.015.

11. Elmaleh D, Shoup T, Bonab A, Takahashi K, Fischman A. Evaluation of 1-deoxy-1-[18F]fluoro-Dmannitol as a brain imaging tracer for measuring osmotic disruption following cancer therapy. $\mathrm{J}$ Nucl Med. 2014;55:1123-1123.

12. Hara T, lio M, Tsukiyama T, Yokoi F. Measurement of human blood brain barrier integrity using 11Cinulin and positron emission tomography. Eur J Nucl Med. 1988;14:173-6.

13. Li Z-B, Wu Z, Cao Q, Dick DW, Tseng JR, Gambhir SS, et al. The Synthesis of 18F-FDS and Its Potential Application in Molecular Imaging. Mol Imaging Biol. 2008;10:92-8.

14. Zhu W, Yao S, Xing H, Zhang H, Tai Y, Zhang Y, et al. Biodistribution and Radiation Dosimetry of the Enterobacteriaceae-Specific Imaging Probe [18F]Fluorodeoxysorbitol Determined by PET/CT in Healthy Human Volunteers. Mol Imaging Biol. 2016;18:782-7.

15. Werner RA, Ordonez AA, Sanchez-Bautista J, Marcus C, Lapa C, Rowe SP, et al. Novel Functional Renal PET Imaging With 18F-FDS in Human Subjects. Clin Nucl Med. 2019;44:410-1.

16. Tran VL, Novell A, Tournier N, Gerstenmayer M, Schweitzer-Chaput A, Mateos C, et al. Impact of blood-brain barrier permeabilization induced by ultrasound associated to microbubbles on the brain delivery and kinetics of cetuximab: An immunoPET study using 89Zr-cetuximab. J Controlled Release. 2020;328:304-12.

17. Goutal S, Gerstenmayer M, Auvity S, Caillé F, Mériaux S, Buvat I, et al. Physical blood-brain barrier disruption induced by focused ultrasound does not overcome the transporter-mediated efflux of erlotinib. J Controlled Release. 2018;292:210-20.

18. Yao S, Xing H, Zhu W, Wu Z, Zhang Y, Ma Y, et al. Infection Imaging With 18F-FDS and First-in-Human Evaluation. Nucl Med Biol. 2016;43:206-14.

19. Weinstein EA, Ordonez AA, DeMarco VP, Murawski AM, Pokkali S, MacDonald EM, et al. Imaging Enterobacteriaceae infection in vivo with $18 \mathrm{~F}$-fluorodeoxysorbitol positron emission tomography. Sci Transl Med. 2014;6:259ra146. 
20. Dauba A, Delalande A, Kamimura HAS, Conti A, Larrat B, Tsapis N, et al. Recent Advances on Ultrasound Contrast Agents for Blood-Brain Barrier Opening with Focused Ultrasound.

Pharmaceutics. 2020;12:E1125.

21. Okada M, Kikuchi T, Okamura T, Ikoma Y, Tsuji AB, Wakizaka $H$, et al. In-vivo imaging of blood-brain barrier permeability using positron emission tomography with 2-amino-[3-11C]isobutyric acid. Nucl Med Commun. 2015;36:1239-48.

22. Iannotti F, Fieschi C, Alfano B, Picozzi P, Mansi L, Pozzilli C, et al. Simplified, Noninvasive PET Measurement of Blood-Brain Barrier Permeability: J Comput Assist Tomogr. 1987;11:390-7.

23. Jones T, Rabiner EA, PET Research Advisory Company. The development, past achievements, and future directions of brain PET. J Cereb Blood Flow Metab. 2012;32:1426-54.

24. Stegmayr C, Bandelow U, Oliveira D, Lohmann P, Willuweit A, Filss C, et al. Influence of blood-brain barrier permeability on O-(2-18F-fluoroethyl)-L-tyrosine uptake in rat gliomas. Eur J Nucl Med Mol Imaging. 2017;44:408-16.

\section{Figures}

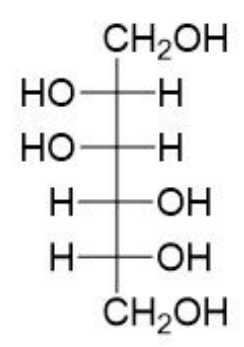

Mannitol

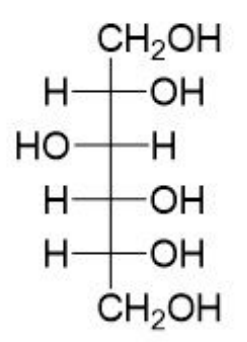

Sorbitol
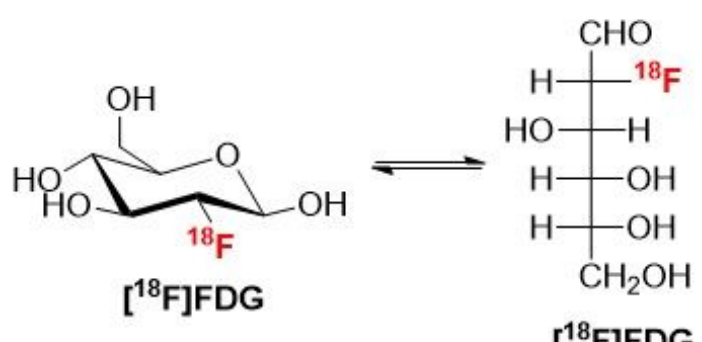

$\left[{ }^{18} \mathrm{~F}\right] \mathrm{FDG}$

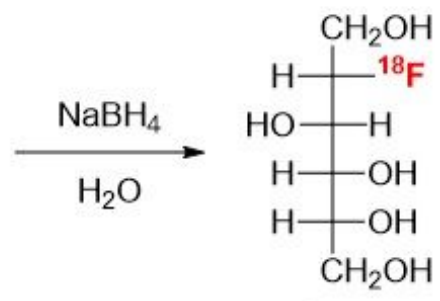

$\left[{ }^{18} \mathrm{~F}\right] \mathrm{FDS}$

\section{Figure 1}

Chemical structures of mannitol, sorbitol, [18F]2-fluoro-2-deoxy-glucose ([18F]FDG) and [18F]2-fluoro-2deoxy-sorbitol ([18F]FDS) 


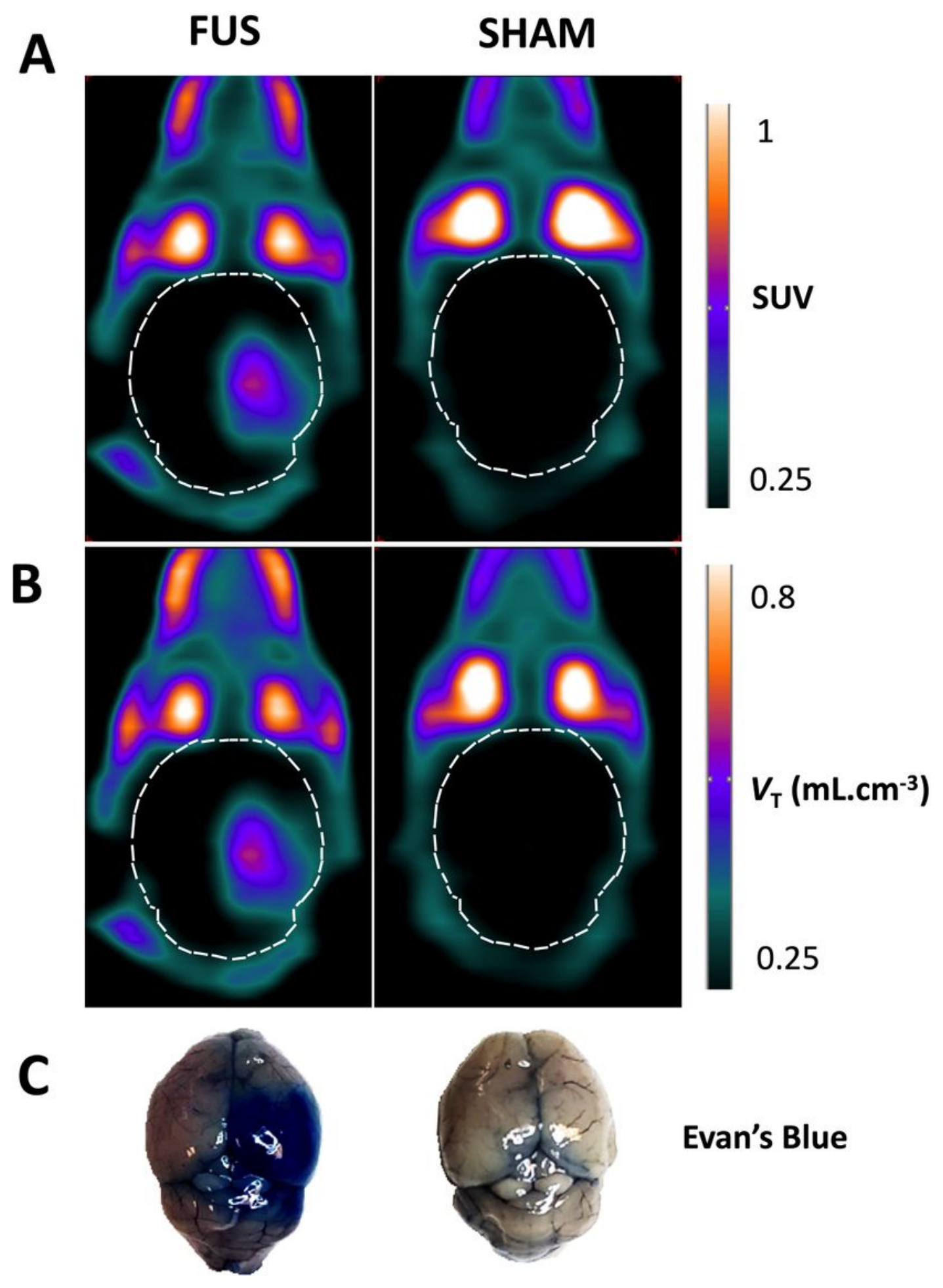

Figure 2

Brain PET images of [18F]2-fluoro-2-deoxy-sorbitol ([18F]FDS) at the intact and disrupted blood-brain barrier (BBB). Representative SUV-normalized brain PET images (sum 30-60 min) of [18F]FDS uptake after (FUS) or without (Sham) hemispheric ultrasound-induced blood-brain barrier disruptions (A). Corresponding parametric VT maps (1-TC model, B) and Evan's Blue extravasation (C) are reported. 


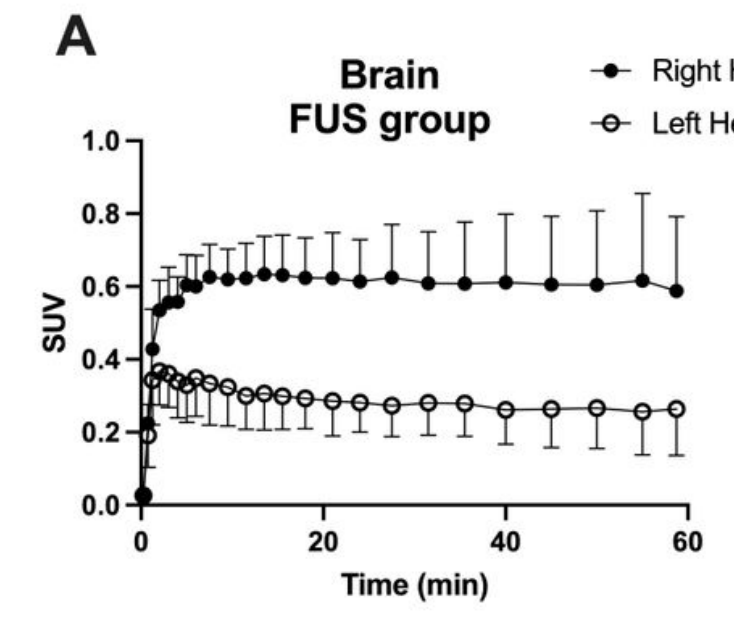

B
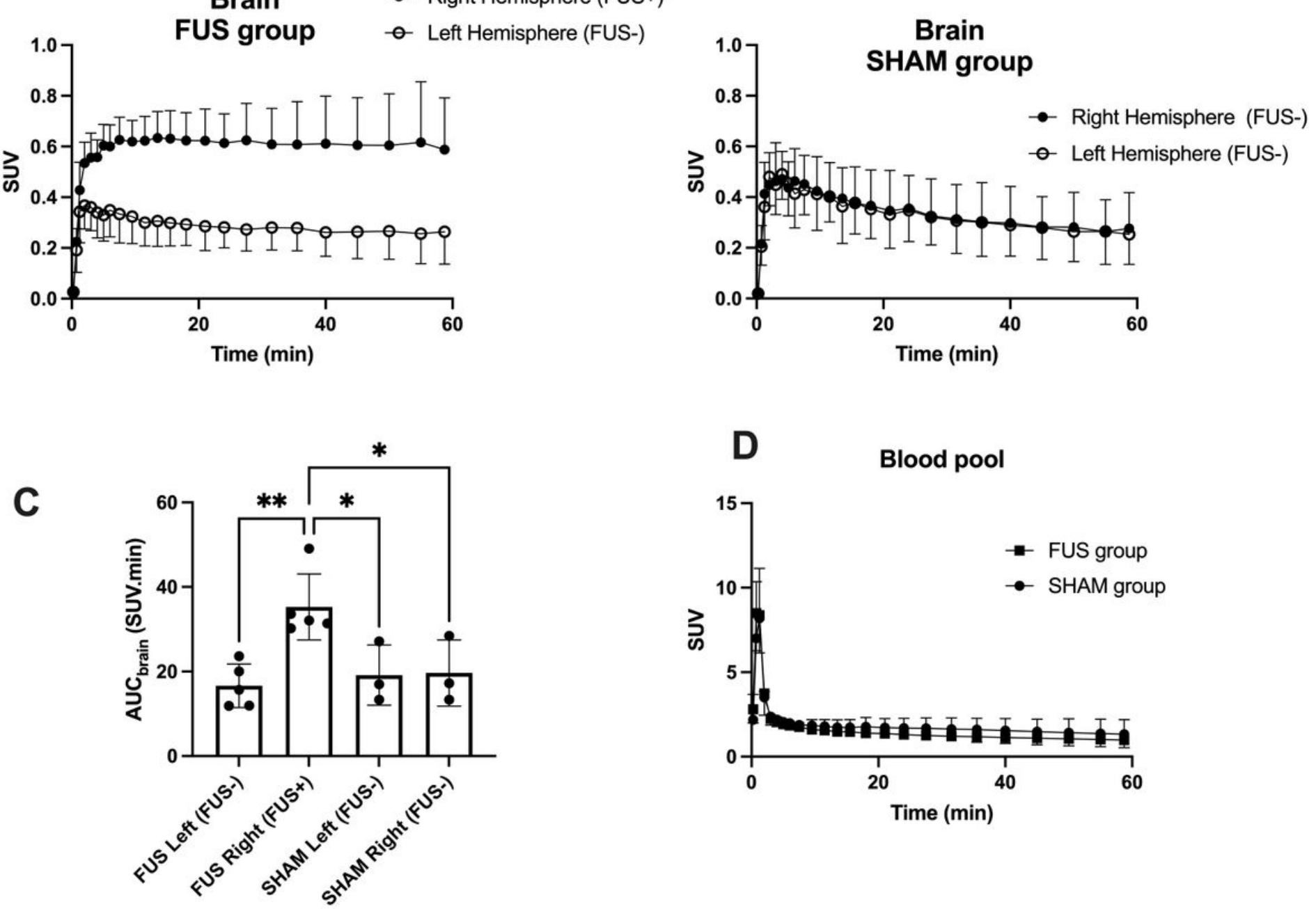

Figure 3

Brain kinetics of [18F]2-fluoro-2-deoxy-sorbitol ([18F]FDS). BBB disruption was obtained in the right brain hemisphere using focused ultrasound (FUS group, A), but not in the Sham group ( $n=3$, in $B)$. In $C$, corresponding brain exposure (AUCbrain) were statistically compared (One-way ANOVA, ${ }^{*} p<0.05$; $\left.{ }^{*} \mathrm{p}<0.01\right)$. Image-derived input functions are shown in D. Data are mean \pm S.D. 

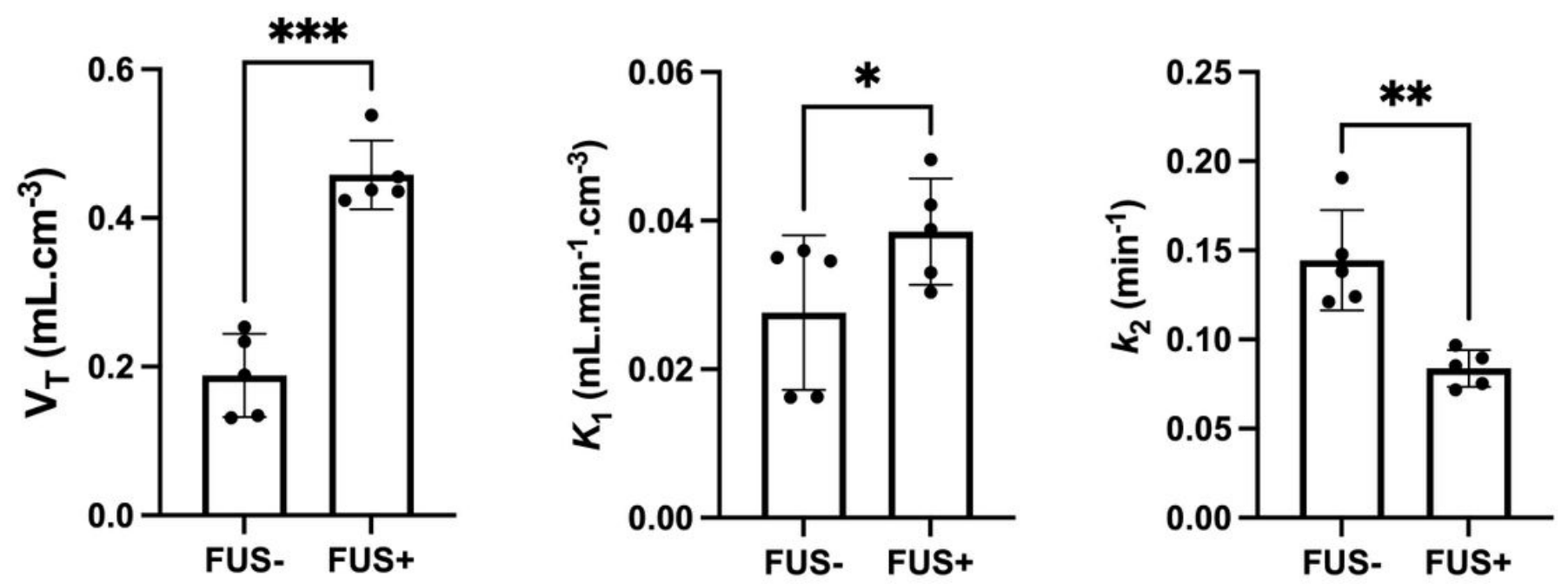

Figure 4

Pharmacokinetic modelling of [18F]FDS brain PET data. Ultrasound-induced BBB disruption (FUS+) was obtained in the right brain hemisphere while BBB in the contralateral brain hemisphere was intact (FUS-). Outcome parameters of the 1-tissue compartment model (VT, K1 and k2) were compared using a paired ttest analysis. Data are reported as mean \pm S.D with individual values $(n=5)$ with ${ }^{*} p<0.05, * * p<0.01$ and $\star \star \star p<0.001$.

\section{Supplementary Files}

This is a list of supplementary files associated with this preprint. Click to download.

- Supplementaryinformation.pdf 